REF. HTHMAR001

\title{
TRANSPORT OF CRITICALLY ILL CHILDREN IN A RESOURCE-LIMITED SETTING
}

Mark Hatherill MBChB, DCH, MRCP(UK), FCPaed(SA)

Paediatric Intensive Care Unit, School of Child \& Adolescent Health, Red Cross Children's Hospital \& University of Cape Town, Cape Town, South Africa.

Correspondence to:

Dr. Hatherill

Institute of Child Health

Red Cross War Memorial Children's Hospital

Klipfontein road

Cape Town

7700

South Africa

Telephone

(SA)(21) 6585354

E-mail

hatheril@ich.uct.ac.za 
The copyright of this thesis vests in the author. No quotation from it or information derived from it is to be published without full acknowledgement of the source. The thesis is to be used for private study or noncommercial research purposes only.

Published by the University of Cape Town (UCT) in terms of the non-exclusive license granted to UCT by the author. 


\section{ABstract}

Background: Transportation of critically ill children by inexperienced personnel may be associated with increased risk of transfer-related adverse events and mortality.

Objective: To audit paediatric intensive care unit (PICU) transfer activity and transfer-related adverse events in a resource-limited setting.

Setting and design: Twenty-two bed regional PICU of a university children's hospital in Cape Town, South Africa. Prospective one-year audit of all children transferred directly to PICU from other hospitals. Data were collected for patient demographics and diagnostic category, referring hospital, transferring personnel, mode of transport, and the incidence of technical, clinical, and critical adverse events. Data are median (interquartile range, IQR). The transfers of 202 children, median age 2.8 months $(1.1-14)$, median weight $3.5 \mathrm{~kg}(2.5-8.1)$ were analysed.

Results: General medical and neonatal surgical conditions comprised $76 \%$ of cases. Children were referred by 38 hospitals, with a median 2 transfers (IQR 2-8, range 126) per institution. Most transfers were performed by paramedic personnel ( $82 \%)$ and via road ambulance $(76 \%)$. One or more technical adverse events, including absent venous access (19\%), inadequate monitoring (13\%), and malposition of the endotracheal tube (25\%), occurred in 36\%. Clinical adverse events, including shock (14\%), hypoxia (13\%), and hypoglycaemia (6\%), occurred in $27 \%$. Critical adverse events, including immediate intubation on admission to PICU (13\%), and cardiac or respiratory arrest (6\%), occurred in $9 \%$. Children retrieved by PICU staff $(10 \%)$, all of whom were from rural centres, had a lower incidence of technical adverse events $(0 \%)$. Children transferred from non-academic hospitals within the greater metropolitan area had a higher incidence of technical (44\%), clinical (39\%), and critical (17\%) adverse events. Mean probability of death was $0.15(95 \%$ CI 0.13 to 0.18 ), with a crude ICU mortality of $17 \%$ and standardised mortality rate of 1.11 $(95 \% \mathrm{CI} 0.83$ to 1.39$)$. There was no significant difference in mortality between academic ( $24 \%)$, metropolitan (11\%), and rural $(15 \%)$ hospitals $(\mathrm{p}=0.12)$.

Conclusion: There is a high incidence of technical, clinical, and critical, transferrelated adverse events. Adverse events occur most commonly in transfers from nonacademic hospitals within the greater metropolitan area. Regional training in advanced life support skills and the deployment of a specialised paediatric ICU retrieval team may reduce the incidence of transfer-related adverse events. Further evaluation would be needed to assess the impact of these measures on ICU mortality. 


\section{INTRODUCTION}

Over the last decade, and particularly since the publication of the Trent-Victoria study, there has been a move towards centralisation of paediatric intensive care in 'developed' countries $1,2,3,4$. This has been accompanied by the development of specialised paediatric retrieval teams (PRT) to undertake the stabilisation and safe transfer of critically ill children from referring hospitals to regional paediatric intensive care units ${ }^{3}$.

It is generally accepted that transfer of unstable critically ill children by inexperienced or non-specialised staff is associated with an unacceptably high incidence of adverse events, and that these events might be associated with increased mortality $5,6,7,8,9$. Proponents of a specialised PRT might argue that a standing team of trained retrieval personnel, with experience of multiple patient transfers, who are familiar with their equipment and the retrieval 'environment', are best equipped to undertake transfers to a paediatric intensive care unit (PICU) $3,4,7,10$.

There is evidence to support that view, in that PRT's have some impact on severity of illness, and experience fewer adverse events during transfer ${ }^{8,10}$. There might also be some advantage to resource allocation, in that a standing regional PRT may benefit from the 'do more, get better' effect". Equally, district hospitals, which might be less able to lose staff, would no longer have to release senior or experienced personnel to undertake patient transfers.

However, it should be noted that proponents of the PRT system stand to gain the additional equipment, funding, and both medical and nursing staff, which would be required to provide a standing service ${ }^{11}$. It is also not clear whether the establishment of a PRT produces the expected reduction in mortality ${ }^{11}$. It may be that the benefit of the PRT system is limited to perceived easier access to the regional PICU, with a consequent increase in external referrals, and flow of funds. It might even be argued that the PRT-Centralised PICU system results in deskilling of referring hospital staff in resuscitation, stabilisation, and transportation ${ }^{12}$.

These questions highlight the need for careful evaluation of regional requirements for paediatric intensive care, and in particular, ongoing assessment of transport systems 
for critically ill children ${ }^{13}$. These issues have been addressed in the context of 'developed' countries, where resources for the establishment of a PRT are more readily available, but there is no literature to guide policy in regions where healthcare resources are limited. The requirements of critically ill children in 'developing' countries, with the additional problems of distance, infrastructure, and perhaps a fragmented, underfunded, and ill-equipped, healthcare system, may be very different.

This study is a prospective audit of the transportation of critically ill children for intensive care at the PICU of a university children's hospital in Cape Town, South Africa. This PICU has provided centralised regional intensive care for many years, but no formal system of referral and transfer exists. Paramedic personnel have traditionally transported critically ill children, and limitation of resources has precluded the establishment of a regional PRT.

The aim of this study was to inform policy for optimal transportation of critically ill children in this healthcare region, and in other 'developing' countries. We might then make recommendations for the allocation of scarce healthcare resources, based on an analysis of regional transfer activity and transfer-related adverse events.

\section{SETTING AND DESIGN}

The PICU provides 22 (18 intensive care and 4 high dependency) of the 30 dedicated PICU beds available in the Western Cape province of South Africa. It is staffed by 3 full-time paediatric intensivists and offers a multi-disciplinary service for critically ill children in metropolitan Cape Town, the Western Cape, and occasionally further afield. It provides care for critically ill children with general medical conditions, as well as those under the care of the general surgical, neurosurgical, cardiothoracic, cardiology, gastroenterology, oncology, nephrology, and neurology paediatric subspecialities.

All children transferred from other hospitals directly to the PICU were eligible for inclusion in the study. Children transferred via other departments within the hospital, such as the trauma unit, medical emergency unit, or radiology department, regardless of the duration of their stay, were not included in the study. 
The duration of the study was 1 calendar year $\left(1^{\text {st }}\right.$ November $2000-31^{\text {st }}$ October 2001). All data were collected, within 24 hours of admission, from the transfer record, the nursing admission record, and the medical admission record. Where these records were incomplete or unclear, data were clarified by brief discussion with the transferring and/or admitting staff.

The following patient and hospital data were collected: age, weight, probability of death predicted by the Paediatric Index of Mortality (PIM) score, observed mortality, diagnosis, time of referral, time of admission to PICU, transferring hospital, transferring personnel, and mode of transport ${ }^{14}$.

In order to exclude children with a low mortality risk, who may not have required PICU referral, transfers of children with less than $1 \%$ probability of death predicted by PIM were not analysed further.

Diagnoses were categorised according to type of illness as medical, general surgical, trauma (including burn injury), cardiac, neonatal surgical (excluding cardiac), and others. Time of referral was defined as the time of telephonic request for admission, or if an elective referral (eg. for peri-operative care), as the time of request for transport to attend the referring hospital.

Referring hospitals were categorised according to type and geographic area as academic, metropolitan, and rural. Academic hospitals were defined as hospitals within the greater Cape Town metropolitan area (within 45 kilometres) with 24-hour paediatric registrar cover. Two tertiary teaching hospitals and two satellite teaching hospitals, all of which provided both neonatal and general paediatric facilities, were included in this category.

Metropolitan hospitals were defined as those (both private sector and government sector) within the greater metropolitan area, but without 24-hour paediatric registrar cover. Nineteen clinics and hospitals were included in this category.

Rural hospitals were defined as district or regional hospitals serving towns beyond the greater metropolitan area, ie. the term 'rural' does not refer only to small hospitals in a 
country setting. Fifteen hospitals were included in this category, the furthest of which is $1200 \mathrm{~km}$ distant.

Transferring personnel were categorised as either PICU or non-PICU staff. PICU staff were either paediatric, anaesthetic, or paediatric surgical registrar trainees, drawn from the rotating staff complement of the PICU. Although they would be proficient in core paediatric critical care skills, they received no formal training in transfer medicine.

Non-PICU staff were either referring hospital doctors, or paramedics. The term 'paramedic' does not refer only to personnel with advanced training in life support skills, but includes all paramedical ambulance support staff. Nursing staff did not undertake unaccompanied transfers.

Mode of transport was categorised as road ambulance, helicopter, or fixed-wing aircraft. Both helicopter and fixed-wing transfers were staffed by experienced paramedics with advanced training in life support skills. PICU medical staff might be asked to retrieve unstable patients who required transfer from more distant rural hospitals by fixed-wing aircraft.

At the time of referral, the referring hospital staff would routinely be given advice on resuscitation, stabilisation, and precautions (such as elective intubation) to be taken prior to transfer.

\section{TECHNICAL ADVERSE EVENTS}

The following data were collected: oxygen saturation monitoring, ECG monitoring, blood pressure monitoring, equipment malfunction, type of venous access, whether venous access was functional or not, endotracheal tube (ETT), route of ETT (oral or nasal), and placement of ETT.

The ETT was judged to be misplaced if on admission to PICU, clinical examination suggested intubation of the right main bronchus and the ETT length at the mouth / nose was greater than suggested for age / weight, or if chest radiograph (CXR) demonstrated the tip of the ETT at the carina or in the right main bronchus, or if 
clinical examination and / or direct laryngoscopy demonstrated that the ETT was sited in the pharynx or oesophagus.

A technical adverse event was defined as one or more of the following: no record of monitoring equipment in place $a b$ initio, malfunction of monitoring or other equipment (eg. oxygen supply), failure to place venous access ab initio, loss of venous access due to displacement or blockage, or malposition of the ETT.

\section{Clinical adverse EVEnTS}

The following data were collected: shock (defined as poor perfusion or hypotension for age, requiring either fluid or inotropic support), use of inotropic support, hypoxia (defined as oxygen saturation $<80 \%$ ), or hypoglycaemia (defined as blood glucose $<$ $2.5 \mathrm{mmol} / \mathrm{L}$ ).

A clinical adverse event was defined as one or more of the following: shock, hypoxia, or hypoglycaemia, occurring either during transfer, or present on admission to PICU.

\section{CRITICAL ADVERSE EVENTS}

The following data were collected: requirement for emergency intubation due to impending collapse on admission to PICU, or occurrence of a cardiac, cardiorespiratory, or respiratory arrest, either during transfer or on admission to PICU.

A critical adverse event was defined as emergency intubation and / or an arrest as above.

Probability of death predicted by PIM was calculated for group comparison, and reported as mean with $95 \%$ confidence intervals $(95 \% \mathrm{CD})$. The standardised mortality ratio (SMR), with $95 \% \mathrm{CI}$, was calculated from the ratio of observed to expected deaths $^{15}$.

Descriptive data are reported as $\mathrm{n}(\%)$, or median (interquartile range) (IQR). Data were analysed using the Fisher's Exact or Chi-squared tests, or by analysis of variance, for parametric and non-parametric data where appropriate. Data were analysed using Analyse-it statistical software (Analyse-it, UK). 


\section{DEMOGRAPHICS}

One thousand two hundred children were admitted to the PICU over the 1-year study period, of whom 234 (20\%), median age 2.7 months (IQR 1.1 - 13), and median weight $3.5 \mathrm{~kg}$ (IQR $2.3-8.1$ ) were transported directly from other institutions.

The 32 patients with less than $1 \%$ risk of mortality predicted by the PIM score, all of whom survived to ICU discharge, were excluded. This group included 18 neonates (56\%) admitted pre-operatively for neonatal surgical conditions.

Two hundred and two children (17\%), median age 2.8 months (IQR $1.1-14)$ and median weight $3.5 \mathrm{~kg}$ (IQR $2.5-8.1$ ) were analysed further.

\section{RESULTS}

Demographic and transfer data for the 202 children transferred to PICU are detailed in Table 1.

\section{PATIENT AND HOSPITAL PROFILE}

Type of illness was predominantly general medical and neonatal surgical ( $76 \%$ of all transfers). Thirty-eight hospitals referred children to PICU, with a median of 2 referrals per hospital (IQR $2-8)$, (range $1-26)$.

\section{TRANSPORT PROFILE}

PICU staff carried out only $10 \%$ of transfers, with the majority ( $82 \%$ ) being undertaken by paramedics. Transportation was by road ambulance in $76 \%$ of cases. Median time from referral to admission to PICU was 3.5 hours (2-6).

\section{TECHNICAL ADVERSE EVENTS}

No venous access was sited in $6 \%$ of children. When venous access was obtained, it consisted of a single intravenous cannula in $79 \%$. Venous access was lost due to dislodgement or blockage in $13 \%$. In total, no functional venous access was available in $19 \%$ of children. 
No monitoring of ECG, pulse oximetry, or blood pressure, was performed during transfer in $11 \%$. Technical failure occurred in $2 \%$, such that inadequate monitoring occurred in $13 \%$ of children.

Fifty-eight per cent $(n=118)$ of children were intubated and ventilated for transfer. The ETT was placed via the oral route in $63 \%$, and via the nasal route in $37 \%$. The ETT was malpositioned in $25 \%$ of intubated children. The ETT was sited in the oesophagus in $6 \%$. Malposition of the ETT was not related to the route of intubation, being $30 \%(n=22)$ in patients intubated orally, and $18 \%(n=8)$, in patients intubated nasally $(\mathrm{p}=0.24)$.

One or more technical adverse events occurred in $36 \%$ of children $(n=72)$.

\section{CLINICAL ADVERSE EVENTS}

Shock and hypoxia were the most common clinical adverse events. Fourteen per cent of children $(n=28)$ were shocked, $13 \%(n=27)$ developed hypoxia, and $6 \%(n=12)$ were hypoglycaemic.

One or more clinical adverse events occurred in $27 \%$ of children $(n=54)$. There was a trend for clinical adverse events to occur more commonly in association with technical adverse events $(n=25,35 \%$, compared to $n=29,22 \%$ ), but this trend did not reach statistical significance $(p=0.07)$.

\section{CRITICAL ADVERSE EVENTS}

Of the 84 children who were not intubated for transfer, $13 \%(n=11)$ required immediate resuscitation, intubation, and ventilation, on admission to PICU. Six per cent of all children $(n=13)$ suffered a cardiac, respiratory, or cardio-respiratory arrest, either during transfer or on admission to PICU.

One or more critical adverse events occurred in $9 \%$ of children $(n=18)$.

\section{TRANSFER PERSONNEL}

Data comparing transfers by PICU and non-PICU personnel are detailed in Table 2. Transfers undertaken by PICU staff were of longer duration (median 9 hours 
compared to 3 hours), and all by fixed-wing aircraft from rural hospitals. These children did not differ in age, weight, or type of illness from those transferred by nonPICU staff.

There was a trend for children transferred by PICU personnel to be intubated more frequently prior to transfer ( $80 \%$ compared to $56 \%$ ). These children were also less likely to have a misplaced ETT ( $0 \%$ compared to $16 \%)$, less likely to experience any technical adverse event ( $0 \%$ compared to $40 \%)$, and more likely to receive inotropic support ( $20 \%$ compared to $4 \%$ ).

There was no difference in the incidence of clinical or critical adverse events between the 2 groups of personnel.

\section{REFERRING HOSPITAL}

Data comparing transfers from academic, metropolitan, and rural hospitals are detailed in Table 3.

Children transferred from metropolitan hospitals were older (median age 6.7 months, weight $5.7 \mathrm{~kg}$ ), and those from academic hospitals younger (median age 1.2 months, weight $2.9 \mathrm{~kg}$ ), than children transferred from rural institutions.

Children transferred from metropolitan hospitals had a higher prevalence of general medical conditions (79\%), and those from academic hospitals had a higher prevalence of neonatal surgical conditions (45\%).

PICU staff retrieved $33 \%$ of children transferred from rural hospitals. A medical doctor accompanied only $6 \%$ of transfers from academic hospitals. All transfers from academic hospitals were via road ambulance, as were the majority (84\%) of metropolitan transfers. The duration of transfer was longer in the case of children transferred from rural hospitals (median 6.3 hours), and these children were more likely to be intubated and ventilated (69\%).

Children transferred from metropolitan hospitals were more likely to experience a technical adverse event ( $44 \%$ compared to $23 \%$ ), such as a misplaced ETT ( $26 \%$ 
compared to $10 \%$ ), than children from a rural hospital. Children transferred from metropolitan hospitals were also more likely to experience either a clinical adverse event (39\%), such as shock ( $23 \%$ ) or hypoxia ( $20 \%)$, or a critical adverse event $(17 \%)$.

\section{OUTCOME AT ICU DISCHARGE}

There were 168 survivors and 34 nonsurvivors, with a crude mortality rate of $17 \%$. Mean probability of death was 0.15 (95\% CI 0.13 to 0.18 ) with an SMR of $1.11(95 \%$ CI 0.83 to 1.39). Two children had a cardiac arrest and died prior to arrival at PICU, one child while still at the referring hospital, and one child while being transferred by PICU staff via fixed-wing aircraft.

Demographic and transfer data for survivors and nonsurvivors are detailed in Table 4. Survivors and nonsurvivors did not differ in age, weight, type of illness, or duration of transfer.

The incidence of technical and critical adverse events did not differ according to survival. There was a trend for clinical adverse events to occur more frequently in nonsurvivors ( $41 \%$ compared to $24 \%$ ), but this difference did not reach statistical significance $(\mathrm{p}=0.07)$.

Severity of illness and outcome data are shown in Table 5.

Non-PICU staff transferred the majority of nonsurvivors, but since non-PICU staff carried out $90 \%$ of all transfers, the difference in crude mortality rate ( $20 \%$ compared to $16 \%)$ was not significant $(\mathrm{p}=0.89)$.

There was no significant difference in crude mortality between academic, metropolitan, and rural hospitals ( $24 \%, 11 \%$, and $15 \%$ respectively), $(\mathrm{p}=0.12)$.

The SMR was higher in children transferred by PICU staff, and lower in children transferred from metropolitan hospitals, but the $95 \%$ confidence intervals are wide and overlapping. 


\section{Discussion}

This study describes the transfer activity of a regional PICU in a resource-limited setting. The Western Cape region does not lack a centralised healthcare infrastructure for critically ill children, in that it already provides 27 PICU beds per million children, based on the estimated $28 \%$ of the 4 million population who are under the age of 15 years (www.cmc.gov.za). However, the region lacks a formal system of transport for children who become critically ill. In order to establish such a service, funding would have to be sought in the face of existing budgetary constraints, to purchase and maintain equipment, and to employ and train additional skilled personnel.

The majority of the regional transfer workload derives from general medical and neonatal surgical patients. This may reflect the dual role of the receiving institution, both as a district referral centre for general paediatrics, and as a tertiary regional referral centre for neonatal and paediatric surgery.

The lack of a regional PRT, and the perceived inability of metropolitan and rural hospitals to release medical staff to accompany patients, may have resulted in the majority of critically ill children $(82 \%)$ being transferred by paramedic staff by default. However, not all paramedic personnel have had training in paediatric advanced life support to equip them to perform this task. It follows that many of the deficiencies highlighted are those of the 'system', rather than of a particular group of transport personnel.

We have demonstrated a high incidence of preventable technical adverse events ( $36 \%$ ), which is comparable to that previously reported for non-specialised transport teams in 'developed' countries ${ }^{5,6,7,8}$. Given that all these children had more than $1 \%$ probability of death, the high incidence of failure to ensure adequate venous access, monitoring of vital signs, and correct placement of the ETT, highlights an area of practice that may be improved upon.

Both clinical and critical adverse events occurred frequently, which again is comparable to the findings of previous studies ${ }^{5,6,7}$. It is of particular concern that $13 \%$ of non-intubated children required immediate intubation on admission to the PICU, 
suggesting either inadequate stabilisation, or failure to recognise the severity of illness, prior to transfer.

It has been demonstrated that a PRT increases the number of clinical interventions prior to transfer, and this is supported by our own study ${ }^{10}$. Children transferred by PICU personnel were more likely to be intubated and receive inotropic support, and less likely to experience an adverse technical event. However, these technical improvements did not translate into a reduction in clinical or critical adverse events. This may be due to the small numbers in this patient group, but others have reported similar findings ${ }^{8}$. Since PICU staff retrieved only $10 \%$ of all children transferred, it became clear during the course of the study that one of the original objectives, an outcome analysis of PICU and non-PICU transfers, would not be possible due to the low statistical power.

It appears that the majority of transfer activity takes place within the greater metropolitan region, by road ambulance, accompanied by paramedic personnel. It follows that it is this category of patients who might derive the maximal benefit from a reorganisation of the transport 'system', and closer analysis of transfer activity from these centres is warranted.

There are clear differences in the type of patient transferred from academic and nonacademic metropolitan institutions. Children referred from academic centres were younger, smaller, and more likely to have a neonatal surgical condition, such as congenital bowel obstruction or necrotising enterocolitis (data not included). By contrast, children from metropolitan hospitals were older children with general medical conditions, such as pneumonia or gastroenteritis.

The fact that PICU staff retrieved no children from the greater metropolitan area makes interpretation of the data more straightforward. However, the small number of doctor-accompanied transfers from nearby academic hospitals, which have 24-hour paediatric registrar cover, raises the question of whether these children would not have been optimally transferred by medical personnel. There was a high incidence of technical adverse events, especially failure of monitoring, in this patient group, but again this did not result in a higher incidence of clinical or critical adverse events. In 
fact, these events occurred less commonly in children from academic institutions, which suggests appropriate clinical assessment of the pre-transfer severity of illness.

By contrast, children transferred from non-academic metropolitan hospitals also had a high incidence of technical adverse events, particularly misplacement of the ETT, but these technical problems were often associated with physiological deterioration. More than one third of these children experienced a clinical adverse event, with both shock and hypoxia occurring frequently. Children transferred from metropolitan hospitals also had the highest incidence of critical adverse events. These problems should be viewed in the context of the limited resources available. These institutions are often small, they may have no on-site paediatric or anaesthetic service, and in some cases a single junior medical doctor provides after-hours care for the entire hospital.

Although the study is not powered to detect differences in sub-group mortality, it may be useful to compare severity of illness and outcome. The standardised mortality rate of 1.11 suggests that the PICU outcome of these children is comparable to that predicted by a 'developed' world risk model (PIM) ${ }^{14}$. It should be noted that this model is almost certainly outdated, with many centres performing better than predicted by PIM, and that it has not been calibrated for this patient population ${ }^{16,17}$.

In fact, it is not clear whether the PIM-based SMR is an appropriate tool to assess outcome after patient transfer in this study. Since PIM utilises first PICU contact data, the SMR is designed to evaluate the outcome of subsequent intensive care ${ }^{14}$. Therefore, the model does not allow differentiation between inherent severity of illness, and subsequent deterioration due to sub-optimal transport. A pre-transfer risk model may be more suited to evaluation of different modes of transfer to PICU ${ }^{6}$.

Despite the small numbers in patient sub-groups, children retrieved by PICU staff had a lower risk of mortality. This may reflect the fact that all these children were referred from distant centres and would usually have been stabilised, often in an adult ICU, by the time of transfer. These patients would have been transferred for 'ongoing', rather than 'acute' intensive care, although data on pre-referral duration of hospital stay was not formally collected. This factor may have influenced the higher SMR in these children, although it should be noted that the confidence intervals are wide. 
There was no significant difference in either risk of mortality, or crude mortality, between referral centres. The differences in SMR suggest a worse outcome for children from academic, compared to metropolitan referring hospitals. At first glance, this is the opposite of what we might expect, given the higher incidence of clinical and critical adverse events in the latter group. This apparent contradiction highlights the weaknesses of the PIM-based SMR for analysis of transfer outcomes. It is also relevant that the PIM model has not been validated in the neonatal surgical population that comprises a large proportion of these academic hospital referrals ${ }^{18,19}$. The model would therefore not discriminate between neonatal surgical, and potentially more readily reversible, general medical conditions.

One of the aims of this study was to provide data upon which policy decisions might be made, to improve the safety of transportation for critically ill children. However, any proposed intervention would be in the context of the limited healthcare resources available in the region, a problem common to many 'developing' countries. It is difficult to draw meaningful conclusions about outcome from this study, for the reasons outlined above, but it is possible to make recommendations based on the incidence and pattern of adverse events.

\section{ACADEMIC HOSPITALS}

Simple pre-transfer technical interventions, such as ensuring adequate monitoring of vital signs, and functional venous access, are inexpensive, and might be expected as the norm at academic institutions that provide tertiary paediatric care. It should be noted from the data, that these measures might not have a great impact on mortality in this group, due to the nature of the underlying illness. A brief pre-transfer checklist may help identify unstable patients who would need to be accompanied by a medical doctor from that institution ${ }^{3}$.

\section{METROPOLITAN HOSPITALS}

The data suggest that it is children referred from these hospitals who have the highest incidence of adverse events (technical, clinical, and critical), and therefore it is this patient group that might benefit most from interventions to improve the quality of transfer to PICU. 
Again, simple pre-transfer interventions would be effective in reducing the high incidence of technical adverse events, and at least allow recognition of, and the capacity to respond to, physiological deterioration en route. The high incidence of clinical and critical adverse events suggests a failure to recognise severity of illness and the potential for clinical deterioration, and a failure to pre-empt such deterioration by stabilisation prior to transfer ${ }^{6}$.

It has been suggested that many of the clinical interventions required to stabilise a sick child for transfer, particularly airway intubation, but also venous access and fluid resuscitation, are not exclusively the province of PICU personnel ${ }^{20}$. There is a strong case for ensuring that all medical personnel in this region, who provide in-patient or emergency care for infants and children, should be trained in both neonatal and paediatric life support skills ${ }^{20,21}$. The Advanced Paediatric Life Support (APLS) course would be an appropriate vehicle for this intervention, and might be provided as an ongoing programme to regional medical staff, with minimal equipment or infrastructure costs. Improved resuscitation at the district hospital level might even lead to a decrease in the number of children needing to be referred for intensive care.

Undoubtedly, there would be critically ill children who remain unstable despite appropriate resuscitation measures, or who require a high-level of ongoing support, and it is these children who might benefit from the availability of a $\mathrm{PRT}^{10}$. A standing PRT, with training in critical care and transport medicine, could be deployed rapidly over the short distances $(<45 \mathrm{~km})$ from the regional PICU. The argument that a PRT would result in deskilling of referring hospital staff does not apply, since only two PICU transfers occurred per hospital per year ${ }^{12}$.

From the data, a PRT would be expected to have an impact on the high incidence of technical adverse events ${ }^{6,7,8,10}$. Given the association between technical and clinical adverse events in this patient group, a PRT might also reduce the high incidence of physiological deterioration ${ }^{6,7,10}$. However, this is not apparent from the current data, and further prospective studies would be required to confirm this hypothesis. 


\section{RURAL HOSPITALS}

Children transferred from rural hospitals are potentially at greatest risk for adverse events, due in part to the longer distances and duration of transfer, and to the relatively 'hostile' transfer environment in the case of helicopter and fixed-wing transfers. These children had a considerable incidence of both technical adverse events, all of which occurred in the non-PICU group, and clinical adverse events. As is the case with metropolitan hospitals, retrieval of all rural hospital referrals by a PRT would be expected to reduce the incidence of technical adverse events, but it remains to be seen whether the incidence of clinical deterioration would fall in parallel $\mathrm{l}^{6,7,8,10}$.

\section{CONCLUSION}

The majority of critically ill children in this region are transported by road ambulance, accompanied by paramedic personnel. The incidence of technical, clinical, and critical adverse events is unacceptably high.

Adverse events are most frequent in transfers from non-academic metropolitan hospitals. Training in advanced paediatric life support skills might reduce the incidence of adverse events in these children.

The establishment of a PRT would be expected to reduce the occurrence of preventable technical adverse events. It would be reasonable to expect a parallel reduction in the incidence of clinical deterioration during transfer. Ongoing prospective evaluation would be necessary to demonstrate the impact of a PRT on clinical adverse events and ICU mortality. 


\section{REFERENCES}

1) Pearson G, Shann F, Barry P, Vyas J, Thomas D, Powell C. Should paediatric intensive care be centralised? Trent versus Victoria. Lancet 1997;349:12131217.

2) Henderson AJ, Garland L, Warne S, Bailey L, Weir P, Edees S. Risk adjusted mortality of critical illness in a defined geographical region. Arch Dis Child 2002;86:194-199.

3) Macrae DJ. Paediatric intensive care transport. Arch Dis Child 1994;71:175178.

4) Mok Q, Tasker R, Macrae D, James I. Impact of specialised paediatric retrieval teams. BMJ 1996;312:119.

5) Bion JF, Wilson IH, Taylor PA. Transporting critically ill patients by ambulance: audit by sickness scoring. BMJ 1988;296:170.

6) Kanter RK, Tompkins JM. Adverse events during interhospital transport: physiologic deterioration associated with pretransport severity of illness. Pediatrics 1989;84:43-48.

7) Barry PW, Ralston C. Adverse events occurring during interhospital transfer of the critically ill. Arch Dis Child 1994;71:8-11.

8) Edge WE, Kanter RK, Weigle CGM, Walsh RF. Reduction of morbidity in interhospital transport by specialized pediatric staff. Crit Care Med 1994;22:1186-1191.

9) Sharples PM, Storey A, Aynsley-Green A, Eyre JA. Avoidable factors contributing to death of children with head injury. BMJ 1990;300:87-91.

10) Britto J, Nadel S, Maconochie I, Levin M, Habibi P. Morbidity and severity of illness during interhospital transfer: impact of a specialised paediatric retrieval team. BMJ 1995;311:836-839.

11) Sajjanhar T, Tibby SM, Hatherill M, Marsh MJ, Murdoch IA. A paediatric retrieval service: its effect on clinical efficacy, activity and resource use in a tertiary paediatric intensive care unit. Clinical Intensive Care 1997;8:178-181.

12) Goldhill DR, Withington PS. Tertiary centres are unproved. BMJ $1998 ; 317: 1320$.

13) Logan S. Commentary: Evaluation of specialist paediatric retrieval teams. BMJ 1995;311:839. 
14) Shann F, Pearson G, Slater A, Wilkinson K. Paediatric index of mortality (PIM): a mortality prediction model for children in intensive care. Intensive Care Med 1997;23:201-207.

15) Rapoport J, Teres D, Lemeshow S, Gehlbach S. A method for assessing the clinical performance and cost-effectiveness of intensive care units: A multicenter inception cohort study. Crit Care Med 1994;22:1385-1391.

16) Parry G, Jones S, Simic-Lawson M. Calibration of the paediatric index of mortality in UK paediatric intensive care units. Arch Dis Child 2002;86:65-69.

17) Tibby SM, Murdoch IA. Calibration of the paediatric index of mortality score for UK paediatric intensive care. Arch Dis Child 2002;86:65-69.

18) Pearson GA, Stickley J, Shann F. Calibration of the paediatric index of mortality in UK paediatric intensive care units. Arch Dis Child 2001;84:125128.

19) Shann F. Where do all the children go? Intensive Care Med 2000;26:6-7.

20) Raffles A. Intensive care provided by local hospitals should be improved. BMJ $1996 ; 312: 120$.

21) Cray SH. London group's findings supported by study in Leeds. BMJ $1996 ; 312: 120$. 


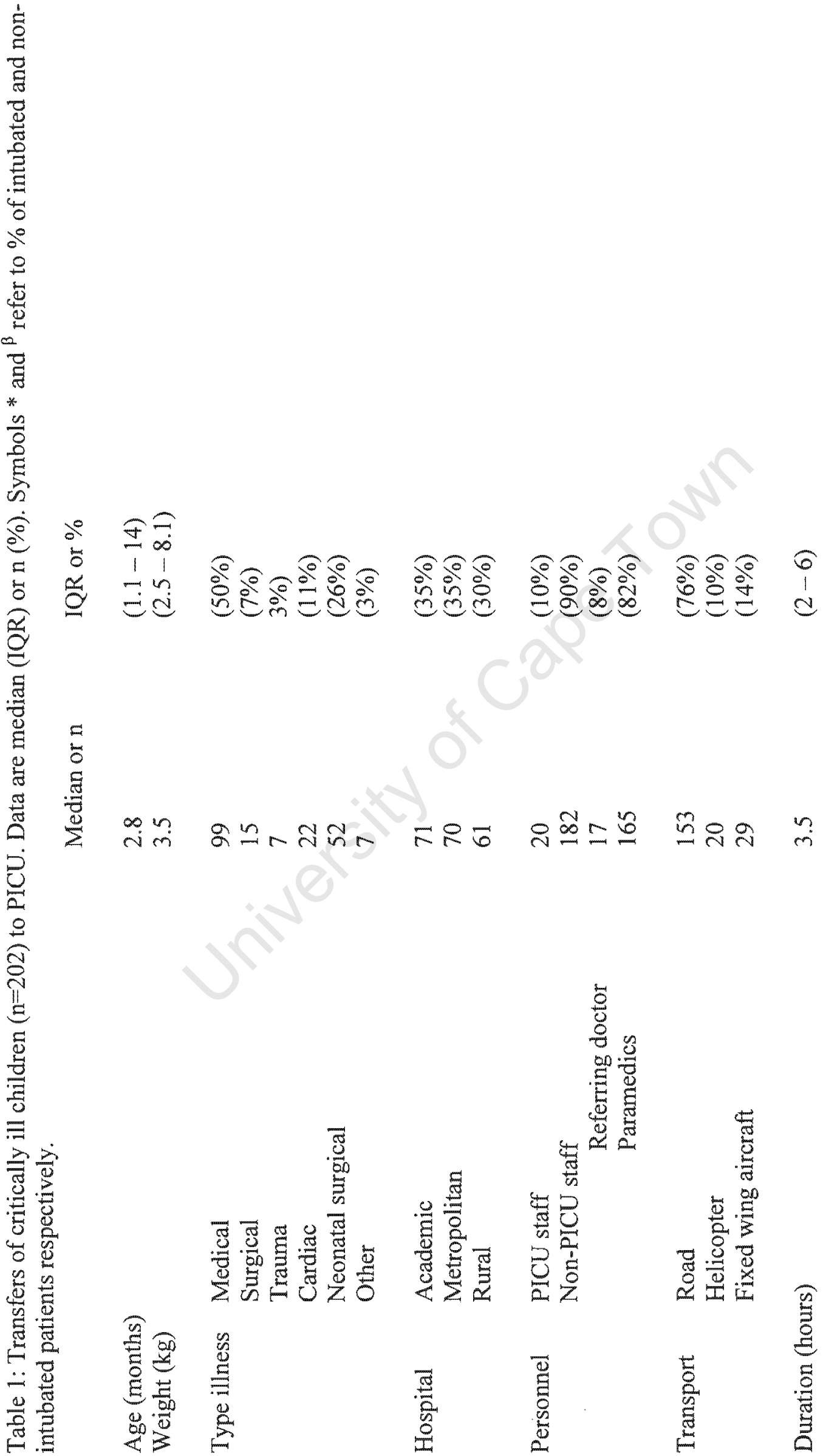


Table 1: continued.

Technical adverse events

No functional venous access (total)

Venous access lost during transfer

Venous access placed

1 peripheral

Central venous

Umbilical venous

Intraosseus

38

26

190

159

19

6

No functional monitoring (total)

No monitoring placed

Technical failure

Intubated for transfer

$$
\begin{aligned}
& \text { Oral } \\
& \text { Nasal } \\
& \text { ETT malpositioned } \\
& \text { ETT oesophageal }
\end{aligned}
$$

Clinical adverse events

Shock

Inotrope

Hypoxia

\section{Critical adverse events}



$=\frac{2}{0} \frac{8}{0}$
$\begin{array}{ll}\overline{8} & \overline{8} \\ 0 & \end{array}$
$E$

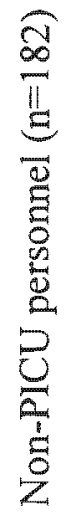

$\underset{1}{\infty}$

용용ㅇㅇ

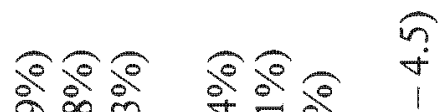

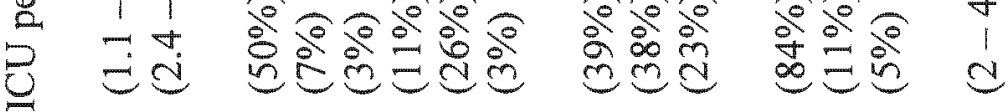

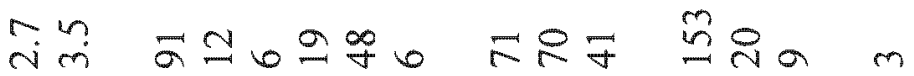

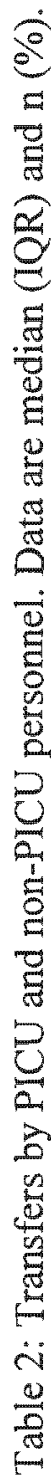

$\widehat{2} \widehat{\Xi}$

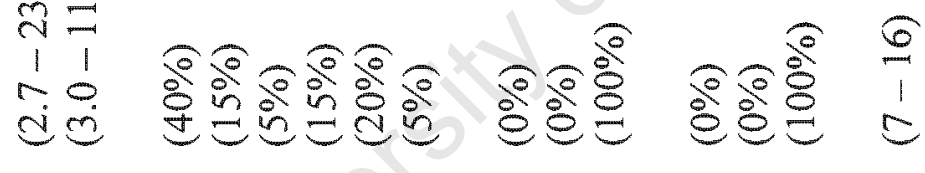

dic

$\stackrel{2}{2}$

ini

$\infty m-m+$

o요 요

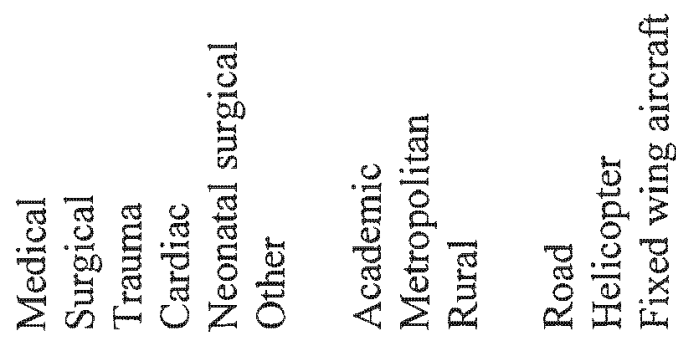

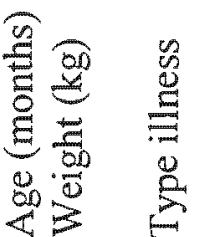

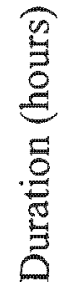


Table 2: continued.

PICU personnel $(n=20)$

Intubated

Technical adverse event

No functional venous access

No functional monitoring

ETT misplacement

Clinical adverse event

Shock

Inotrope

Hypoxia

Hypoglycaemia

Critical adverse event
$16(80 \%)$

$(0 \%)$

$(0 \%)$

$(0 \%)$

$(0 \%)$

$5 \quad(25 \%)$

$3 \quad(15 \%)$

$4 \quad(20 \%)$

$2 \quad(10 \%)$

$1 \quad(5 \%)$

$2 \quad(10 \%)$
Non-PICU personnel $(\mathrm{n}=182)$

$\begin{array}{lll}102 & (56 \%) & 0.06 \\ 72 & (40 \%) & 0.0002 \\ 26 & (14 \%) & 0.11 \\ 26 & (14 \%) & 0.11 \\ 30 & (16 \%) & 0.012 \\ & & \\ 49 & (27 \%) & 1.0 \\ 25 & (14 \%) & 1.0 \\ 8 & (4 \%) & 0.04 \\ 25 & (14 \%) & 0.96 \\ 11 & (6 \%) & 1.0 \\ 16 & (9 \%) & 1.0\end{array}$



。䜌謇
言
囍
言

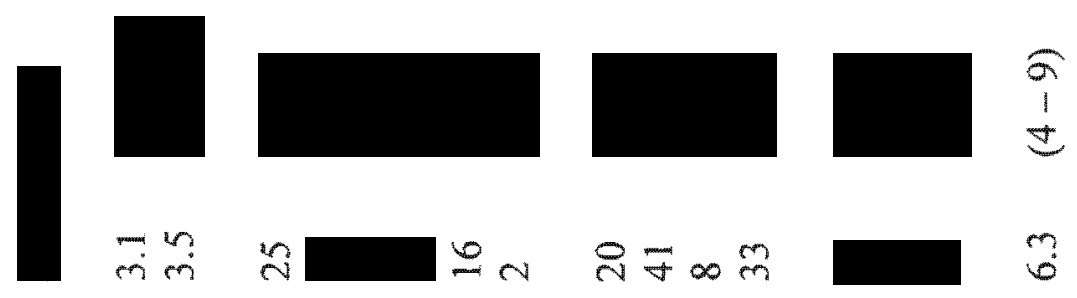

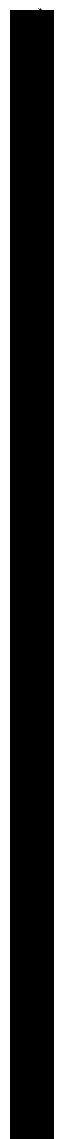

苞

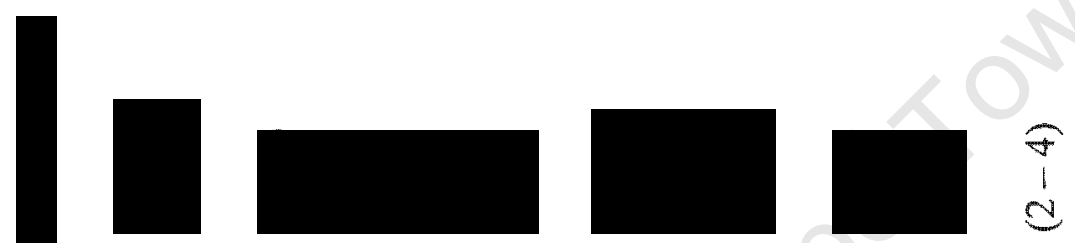

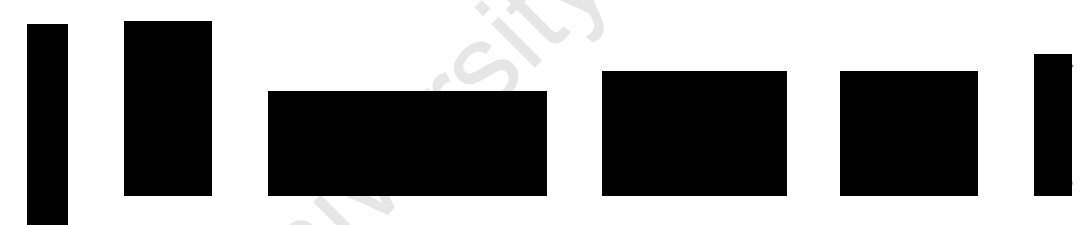

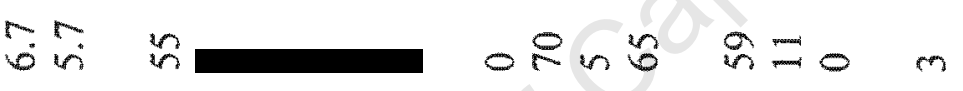

Iี

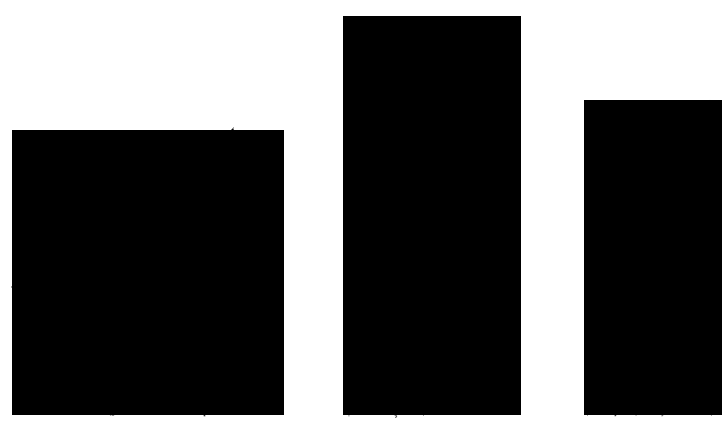

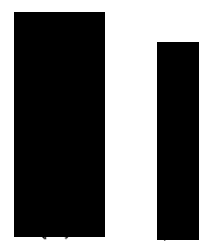

产

홍
$\frac{0}{3}$
롤

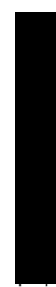


Table 3: continued.

\begin{tabular}{|c|c|c|c|c|c|}
\hline \multirow[b]{2}{*}{ Intubated } & Academic $(n=71)$ & Metropolitan $(n=70)$ & \multicolumn{2}{|c|}{ Rural $(n=61)$} & \multirow{2}{*}{$\begin{array}{l}\mathrm{p} \\
0.006\end{array}$} \\
\hline & $31 \quad(44 \%)$ & $(64 \%)$ & 42 & $(69 \%)$ & \\
\hline Technical adverse event & $27 \quad(38 \%)$ & $(44 \%)$ & 14 & $(23 \%)$ & 0.034 \\
\hline No functional venous access & $8 \quad(11 \%)$ & $(16 \%)$ & 7 & $(11 \%)$ & 0.68 \\
\hline No functional monitoring & $16 \quad(23 \%)$ & $(10 \%)$ & 3 & $(5 \%)$ & 0.007 \\
\hline ETT misplacement & $6 \quad(8 \%)$ & $(26 \%)$ & 6 & $(10 \%)$ & 0.015 \\
\hline Clinical adverse event & $(18 \%)$ & $(39 \%)$ & 14 & $(23 \%)$ & 0.018 \\
\hline Shock & $(7 \%)$ & $(23 \%)$ & 7 & $(11 \%)$ & 0.02 \\
\hline Inotrope & $(6 \%)$ & $(4 \%)$ & 5 & $(8 \%)$ & 0.63 \\
\hline Hypoxia & $(6 \%)$ & $(20 \%)$ & 9 & $(15 \%)$ & 0.04 \\
\hline Hypoglycaemia & $(10 \%)$ & $(6 \%)$ & 1 & $(2 \%)$ & 0.14 \\
\hline Critical adverse event & $(3 \%)$ & $(17 \%)$ & 4 & $(7 \%)$ & 0.009 \\
\hline
\end{tabular}


Table 4: Survivors and nonsurvivors. Data are median (IQR) and n (\%).

\begin{tabular}{|c|c|c|c|c|c|c|}
\hline & & Surv & $\operatorname{ors}(n=168)$ & Non & Irvivors $(n=34)$ & $\mathrm{p}$ \\
\hline Age (month & & 2.8 & $(1-13)$ & 3.1 & $(1.3-24)$ & 0.43 \\
\hline Weight $(\mathrm{kg})$ & & 3.5 & $(2.6-8)$ & 3.8 & $(2.4-11)$ & 0.54 \\
\hline Duration (he & & 3.5 & $(2-6)$ & 3.0 & $(2-4)$ & 0.11 \\
\hline Type illness & Medical & 86 & $(51 \%)$ & 13 & $(38 \%)$ & 0.48 \\
\hline & Surgical & 11 & $(7 \%)$ & 4 & $(12 \%)$ & \\
\hline & Trauma & 5 & $(3 \%)$ & 2 & $(6 \%)$ & \\
\hline & Cardiac & 16 & $(10 \%)$ & 6 & $(17 \%)$ & \\
\hline & Neonatal surgical & 44 & $(26 \%)$ & 8 & $(24 \%)$ & \\
\hline & Other & 6 & $(3 \%)$ & 1 & $(3 \%)$ & \\
\hline Hospital & Acadernic & 54 & $(32 \%)$ & 17 & $(50 \%)$ & 0.12 \\
\hline & Metropolitan & 62 & $(37 \%)$ & 8 & $(24 \%)$ & \\
\hline & Rural & 52 & $(31 \%)$ & 9 & $(26 \%)$ & \\
\hline Personnel & PICU & 16 & $(10 \%)$ & 4 & $(12 \%)$ & 0.44 \\
\hline & Non-PICU & 152 & $(90 \%)$ & 30 & $(88 \%)$ & \\
\hline Transport & Road & 127 & $(76 \%)$ & 26 & $(76 \%)$ & 0.84 \\
\hline & Helicopter & 16 & $(10 \%)$ & 4 & $(12 \%)$ & \\
\hline & Fixed wing aircraft & 25 & $(14 \%)$ & 4 & $(12 \%)$ & \\
\hline Technical ac & yerse event & 63 & $(38 \%)$ & 9 & $(26 \%)$ & 0.30 \\
\hline Clinical adv & rse event & 40 & $(24 \%)$ & 14 & $(41 \%)$ & 0.07 \\
\hline Critical adv & se event & 13 & $(8 \%)$ & 5 & $(15 \%)$ & 0.33 \\
\hline
\end{tabular}




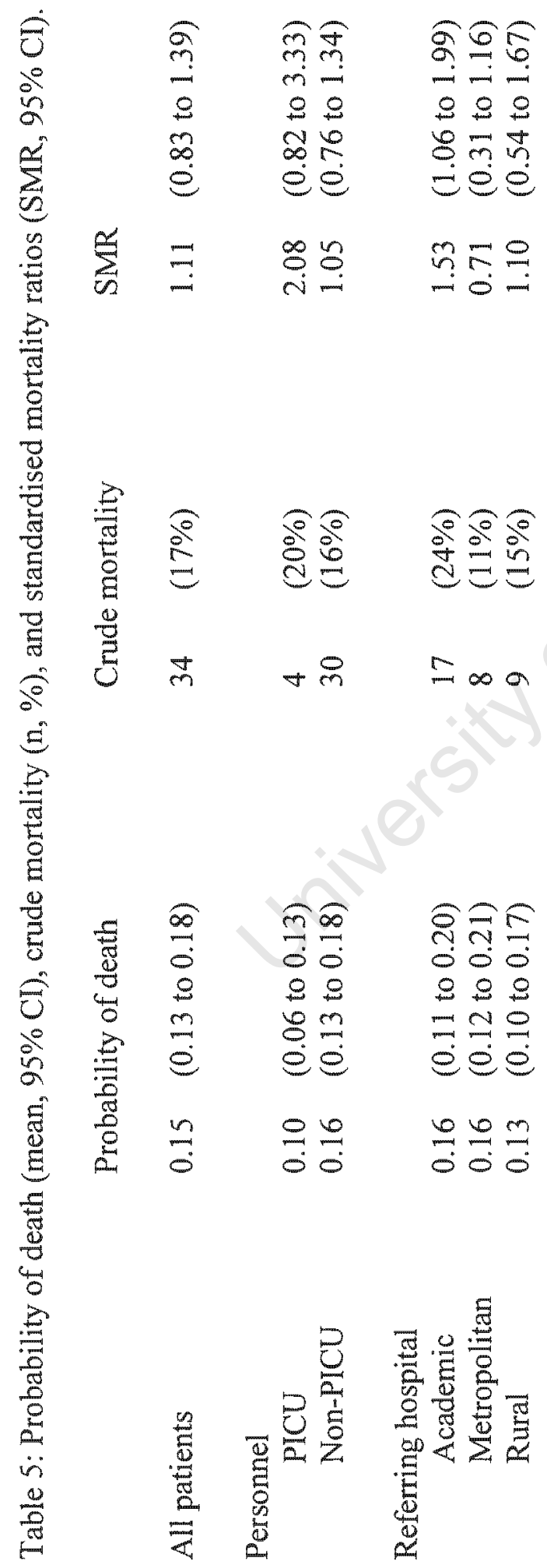

